\title{
Pain Score in Adjustable Strabismus Surgery
}

\author{
Sana Nadeem ${ }^{1}$ \\ ${ }^{1}$ Department of Ophthalmology, Fauji Foundation Hospital, Rawalpindi
}

\begin{abstract}
Purpose: To assess the pain experienced by patients at the time of suture adjustment using topical proparacaine hydrochloride $0.5 \%$ anesthesia, in adjustable suture strabismus surgery.

Study Design: Prospective, interventional study.

Place and Duration of Study: Eye department of Fauji Foundation Hospital, Rawalpindi from May, 2017 to March, 2019.

Methods: A prospective study was carried out to assess the pain experience of patients undergoing suture adjustment under topical proparacaine hydrochloride $0.5 \%$ anesthesia, during routine adjustable squint surgery. All surgeries were performed under general anesthesia with suture adjustment done 1 hour or more after surgery when the effects of general anesthesia had worn off. Horizontal and vertical muscle recessions and resections were included along with inferior oblique surgeries, and transposition procedures. The patients were given the 'Wong-Baker FACES ${ }^{\circledR}$ Pain rating Scale'; along with a 'Numeric Pain Rating Scale' from 0 to 10 (0 signifying 'no pain' and 10 signifying 'worst possible pain') on a proforma. The response of the patients was noted.

Results: Thirty three patients who underwent adjustable strabismus surgery were included in this study. The mean age was $19.1 \pm 11.1$ years. The average number of muscles operated upon for each patient were $2.87 \pm$ 1.08. The patients' response to the 'Wong-Baker FACES ${ }^{\circledR}$ Pain rating Scale' ranged from 0 to 8 , with a mean of $2.03 \pm 1.81$ SD. On the 'Numeric Pain Rating Scale' a similar response was obtained with a mean of $2.0 \pm 1.82$ SD. Augmentation of anesthesia was not needed in any patient.

Conclusion: Adjustment of sutures under topical proparacaine hydrochloride $0.5 \%$ anesthesia after strabismus surgery is a practical, comfortable and safe procedure.
\end{abstract}

Key Words: Adjustable suture, Strabismus, Pain score, Pain scale, Topical anesthesia.

How to Cite this Article: Nadeem S. Pain Score in Adjustable Strabismus Surgery. Pak J Ophthalmol. 2020, 36 (2): 109-113.

Doi: $10.36351 /$ pjo.v36i2.912

\section{INTRODUCTION}

Adjustable suture strabismus surgery was first described in 1907 by Bielchowsky thus allowing the

Correspondence: Sana Nadeem

Assistant Professor, Department of Ophthalmology

Fauji Foundation Hospital, Rawalpindi

Received: September 24, 2019

Accepted: March 15, 2020 surgeon a second chance at realigning the eyes and improving his results. Jampolsky in 1975 described a two-stage adjustable strabismus technique, with the surgery performed under general anesthesia and suture adjustment done later under topical anesthesia, $4-24$ hours after surgery to fine tune the results. ${ }^{1-3}$ Tripathi $^{3}$ et al believe that adjustable strabismus surgery is the procedure of choice for all fit and willing patients. Awadein and Guyton et $\mathrm{al}^{4}$ recommend adjustable sutures in all patients including infants and children as well. 
Topical anesthesia with proparacaine hydrochloride $0.5 \%$ has been a safe and effective tool during the final suture adjustment and has been advocated by many strabismus surgeons. ${ }^{4,5,6}$ Seijas et al recommend topical anesthesia only, for strabismus surgery even without the need of a general anesthetic. ${ }^{7}$

The rationale of our study was to assess the pain score at the time of suture adjustment after strabismus surgery using two scales, in order to find out if it is a comfortable and effective procedure.

\section{METHODS}

A total of 33 patients were included in this study by convenient sampling technique. The study was carried out in the Department of Ophthalmology, Fauji Foundation Hospital, Rawalpindi; a tertiary care teaching hospital affiliated with the Foundation University Medical College. Approval from the ethical committee was taken. Horizontal, vertical and complex strabismus cases were included along with patients with a previous history of strabismus surgery. Myasthenia gravis and uncooperative children less than 7 years of age were excluded.

A detailed ocular assessment was done and bestcorrected visual acuity was documented for every case. Refractive correction was given to the patients before surgery. The prism cover test (PCT) was used to assess the preoperative angle of deviation with the refractive correction in place, for both near and distance in primary gaze position, as well as in $25^{\circ}$ of upgaze (chin down) and $35^{\circ}$ of downgaze (chin up), right and left gaze, and head tilt in case of paralytic strabismus. ${ }^{8}$ In certain cases of sensory strabismus with poor fixation, the Krimsky test was used for analysis of the angle or a pen torch used as a target for near and distance. The distance angle in primary position with the refractive correction in place was considered as the angle of deviation in all cases, and the surgical alignment was sought to correct this angle, although at the time of suture adjustment, both near and distance alignment was corrected. Exception to this was accommodative refractive esotropia, for which the near deviation with distance spectacles in place was considered for correction of the alignment.

The measurements were taken by the operating surgeon and repeated one day prior to the surgery, to obtain maximum cosmesis. Extraocular motility was checked with muscle overaction graded from +1 to +4 and underaction graded from -1 to -5 . Binocular vision and stereopsis were assessed by the Titmus fly test and Worth four dot test in every case. A thorough eye examination was performed including fundus and intraocular pressures. In case of significant oblique overaction, paralytic or vertical strabismus, fundus torsion was also assessed with the indirect ophthalmoscope. All surgeries were performed by the author under general anesthesia. A drop of phenylephrine $10 \%$ (Ethifrin ${ }^{\circledR}$ ) was instilled into the conjunctival sac prior to the surgery in each eye. The fornix approach for strabismus surgery was used in every case. Each muscle was hooked, and then secured with a double armed $6-0$ vicryl (polyglactin 910) absorbable suture, which was passed through the sclera at muscle insertion, or transposed above or below the insertion in case of "A" or "V" patterns, in a 'hang-back' fashion. The muscles placed for adjustable purpose were held in position by Guyton's modification of the sliding noose knot, which was fashioned with a $6-0$ vicryl suture. ${ }^{9}$ The amount of 'hang-back' recession was calculated for each patient using standard tables. ${ }^{10-12}$ The traction suture for holding the sclera for postoperative adjustment was created with ethibond 5-0 in every case. For the nonadjustable recessions, the muscle was tied and allowed to 'hang-back' from its insertion, with the amount of recession calculated as required. For resections, the amount of resection was overcorrected by $2 \mathrm{~mm}$, and allowed to 'hang-back' for this distance, to be adjusted if required postoperatively. At least one muscle was kept on an adjustable sliding noose knot per case; with complex strabismus, all muscles were kept on adjustable sutures.

All patients were assessed for alignment and final adjustment at least 1 hour or more after surgery, in the recovery room, to allow the effects of general anesthesia to wear off. The eyes were anesthetized topically with Alcaine ${ }^{\circledR}$ (proparacaine hydrochloride $0.5 \%$ ) eye drops a few times. The patients were fully conscious at the time of suture adjustment and were not placed on a monitor. However, they were observed for discomfort, syncope or oculocardiac reflex. The patients were assessed with the cover-uncover test at distance and near, with a torch light for distance if the vision was blurred, or a distance readable target; and for near an accommodative target was used. If the alignment was satisfactory, with no movement on cover testing, the sutures were tied off in their existing position, held in place by the sliding noose, which was removable after tying the ends of the muscle sutures. 
Thereafter, the traction knot was cut, and the conjunctiva was sutured with at least one 6-0 vicryl suture. The final tying off point was orthotropia or maximum possible under-correction as required. In cases of exotropia, the goal was orthotropia or mild esotropia. In cases of esotropia, the goal was either orthotropia, if achieved, or slight under-correction. Topical steroid and antibiotic drops and ointment were instilled. No bandage was applied in any case. The next day, the patients were asked about their pain experience by giving them a proforma with two scales; the 'Wong-Baker FACES ${ }^{\circledR}$ Pain rating Scale ${ }^{13}$ ' and a 'Numeric Pain Rating Scale ${ }^{14}$ (NPRS)'.

The Wong-Baker scale is a pain grading scale which was developed by Donna Wong and Connie Baker, depicting a series of 6 faces starting from a happy face with 0 or 'no hurt' to a weeping face at 10 representing 'worst pain imaginable' (Figure 1). This scale was initially developed for children, but nowadays is used for patients 3 years and above. The Numeric Pain Rating Scale is a unidimensional assessment of pain severity in adults and is a segmented numeric version of the Visual analog scale (VAS). It ranges from $0-10$ with 0 representing 'No pain', 5 representing 'Moderate pain' and 10 representing 'Worst possible pain' (Figure 2).

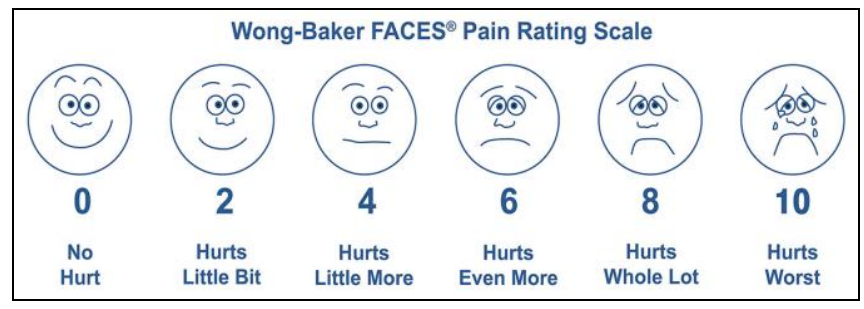

Fig. 1:

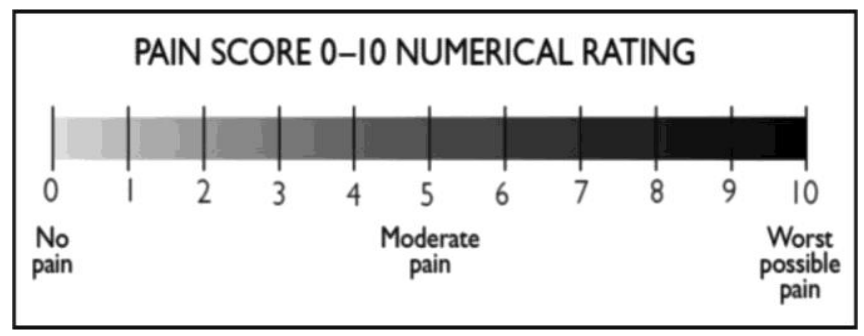

Fig. 2: Numeric Pain Rating Scale.

The patients were explained each scale and asked to point their level of pain on each scale with a finger. The name, age and gender of the patient, type of strabismus, diagnosis, surgery performed and the number of muscles operated upon, were filled on each performa. The results were noted, tabulated and analyzed using SPSS statistics version 20. Frequencies and percentages were calculated for age, gender, type of strabismus, surgical procedure performed, number of muscles and the pain score. Paired t-tests were used to assess the effect of number of muscles operated upon on the pain score.

\section{RESULTS}

A total of 33 consecutive patients with strabismus presenting to us were included in this study. The mean age was $19.1 \pm 11.1$ years (range $7-69$ ). Majority of the patients were female $(81.8 \%)$ and the rest were male $(18.2 \%)$. The deviation type in the majority of the patients was exotropia in $14(42.5 \%)$ cases (Table 1).

Table 1: Type of Deviation.

\begin{tabular}{|lc}
\hline Type of Deviation & Frequency (Percent) \\
\hline Exotropia & $14(42.4)$ \\
Esotropia & $7(21.2)$ \\
Esotropia \& DVD & \\
Exotropia \& Hypertropia & $2(6.1)$ \\
Esotropia \& Hypertropia & $4(12.1)$ \\
Esotropia \& Hypotropia & $1(3)$ \\
Exotropia \& Hypotropia & $3(9.1)$ \\
Horizontal & $2(6.1)$ \\
Horizontal and Vertical & $13(39.4)$ \\
Complex & $4(12.1)$ \\
Horizontal \& Complex & $\epsilon$ \\
Horizontal, Vertical \& Complex & $13(39.4)$ \\
$\Phi$ Dissociated vertical deviation & $2(6.1)$ \\
$€$ Sensory, Monocular elevation & $1(3)$ \\
nystagmus or DVD & \\
\end{tabular}

Table 2 outlines a list of surgeries performed for the primary deviation. The average number of muscles operated upon for each patient were $2.87 \pm 1.08$ (Range 2-6).

The patients' response to the 'Wong-Baker $F A C E S^{\circledR}$ Pain rating Scale' ranged from 0 to 8 , with a mean of $2.03 \pm 1.81 \mathrm{SD}$, signifying 'hurts little bit'. On the 'Numeric Pain Rating Scale' a similar response was obtained with a mean of $2.0 \pm 1.82$ SD (Range $0-$ 8 ), signifying mild discomfort. Only one patient complained of significant pain, rating 8 on both scales, but in this case too, no additional anaesthesia was needed and adjustment was performed successfully. She also had all three muscles on adjustable sutures, 
Table 2: Surgical Procedures Performed for Primary Deviation.

A. Horizontal Muscle Surgery.

\begin{tabular}{|c|c|}
\hline Surgery & Frequency (Percent) \\
\hline $\mathrm{BLRc}^{\mathrm{q}}$ & $8(24.2)$ \\
\hline $\mathrm{BMRc}^{\S}$ & $8(24.2)$ \\
\hline$M R c^{\alpha}+\operatorname{LRs}^{x}$ & $1(3)$ \\
\hline $\mathrm{MRs}^{\emptyset}+\mathrm{LRc}^{\mathrm{H}}$ & $7(21.2)$ \\
\hline $\mathrm{BLRc}^{\mathbb{I}}+\mathrm{MRs}^{\emptyset}$ & $2(6.1)$ \\
\hline $\mathrm{BMRc}^{\S}+\mathrm{LRs}^{\times}$ & $2(6.1)$ \\
\hline $\operatorname{MRc}^{\mathrm{a}}$ & $2(6.1)$ \\
\hline $\mathrm{LRc}^{*}$ & $1(3)$ \\
\hline $\mathrm{LRc}^{\mathrm{H}}+\mathrm{MRs}^{\phi}+\mathrm{MRc}^{\alpha}$ & $1(3)$ \\
\hline None & $1(3)$ \\
\hline
\end{tabular}

II Bilateral recessions

$\S$ Bimedial recessions

$¥ \quad$ Bimedial resections

a Unilateral medial rectus recession

$\times$ Unilateral lateral rectus resection

$\varnothing \quad$ Unilateral medial rectus resection

$\mathrm{H}$ Unilateral lateral rectus recession

* Unilateral lateral rectus recession

B. Vertical Muscle Surgery.

\begin{tabular}{ll}
\hline Surgery & $\begin{array}{c}\text { Frequency } \\
\text { (Percent) }\end{array}$ \\
\hline None & $17(51.5)$ \\
Unilateral IO $\mathrm{IO}^{\alpha}$ myectomy & $4(12.1)$ \\
Bilateral IO ${ }^{\alpha}$ myectomies & $6(18.2)$ \\
$\mathrm{IRc}^{\lambda}+\mathrm{IO}^{\alpha}$ myectomy & $1(3)$ \\
$\mathrm{Bilateral} \mathrm{IO}^{\alpha}$ myectomy $+\mathrm{SR}^{\Omega}$ transposition & $1(3)$ \\
$\mathrm{IRc}^{\lambda}+\mathrm{SRc}^{2}$ & $3(9.1)$ \\
$\mathrm{IRc}^{\lambda}$ & $1(3)$ \\
\hline
\end{tabular}

$\alpha \quad$ Inferior oblique

$\Omega$ Superior rectus

$\Sigma$ Superior rectus recession

$\lambda$ Inferior rectus recession

and this could have contributed to her discomfort. However, no correlation between the number of muscles operated upon and the pain scales was observed. 10 patients $(30.3 \%)$ reported no pain whatsoever, scoring 0 on both scales. No complication of any kind was seen in any case during the suture adjustment procedure.

\section{DISCUSSION}

Adjustable suture strabismus surgery has been done under many different types of anaesthesia. General anaesthesia is preferred for the initial procedure and final suture adjustment is done under topical anaesthesia. Proparacaine hydrochloride $0.5 \%$ ophthalmic solution or proxymetacaine is topical anaesthetic of the aminoester drug group, which antagonizes the voltage-gated sodium channels to alter permeability of neuronal membranes. ${ }^{15}$ Topical anaesthesia has been found to be a safe and effective tool during suture adjustment in strabismus surgery ${ }^{4}$. Sharma et $\mathrm{al}^{5}$ reported that adjustable strabismus surgery under topical anaesthesia was a safe and better option than conventional recession-resection surgery for concomitant Exodeviation. However, Seijas et $\mathrm{al}^{7}$ reported oculocardiac reflex in 3 patients for which atropine was given. They also suggested that monitoring by anaesthetist was necessary because of vagal reflex. None of our patients experienced any complication like that.

Mazow $\mathrm{ML}^{16}$ also described good results following adjustable suture strabismus surgery but stressed upon the importance of good case selection. Similarly $81.8 \%$ success was reported by Park JM et al in adjustable suture strabismus surgery. ${ }^{17}$

Nowadays, many surgeons are striving for strabismus surgery under local anaesthesia only including retrobulbar, peribulbar, subconjunctival and subtenon injections or even topical anaesthesia only. For local anaesthetic injections, although the complications of general anaesthesia are avoided, at least 6 hours are required before adjusting sutures, to allow the effects of the local anaesthetics to wear off. ${ }^{18-21} \mathrm{We}$ prefer general anaesthesia for our strabismus surgeries in all cases, with suture adjustment under topical proparacaine hydrochloride $0.5 \%$ drops only, after 1 hour or more has elapsed after surgery, at which time the patient is fully conscious, able to obey commands and no longer under the effects of the general anaesthetics. This provides an accurate assessment of the residual strabismus.

In our study, we found suture adjustment under topical proparacaine hydrochloride $0.5 \%$ to be safe, comfortable and reliable. All patients reported a good experience, with no or mild pain during suture adjustment, ranging from $0-4$ on both scales, with the exception of one lady who reported significant pain at a rating of 8 on both scales. The number of muscles operated upon did not correlate with excessive pain on the pain scales. Augmentation with other anaesthetic agents was not needed in any case, and neither was a reoperation required for any patient at a later time. No complications were seen during adjustment, and all patients were cooperative and adjusted on the same day. Oculocardiac reflex was not observed in any patient during suture manipulation. Topical 
anaesthesia also saved time compared to the local anaesthesia procedures.

Limitations of this study are small sample size, but still we want to share our thoughts on adjustable strabismus pain score at this time. The findings of our study suggest adjustable strabismus surgery as a good technique for better cosmetic and functional results.

\section{CONCLUSION}

Final suture adjustment under topical proparacaine hydrochloride $0.5 \%$ drops is a safe, effective and comfortable procedure, with minimal pain experienced by patients.

\section{Ethical Approval}

The study was approved by the Institutional review board/Ethical review board.

\section{Conflict of Interest}

Authors declared no conflict of interest

\section{REFERENCES}

1. Nihalani BR, Hunter DG. Adjustable suture strabismus surgery. Eye, 2011 Oct; 25 (10): 1262-1276.

2. Saxena R, Dhiman R. Commentary: Adjustable strabismus surgery. Indian J Ophthalmol. 2019 Feb; 67 (2): 263.

3. Tripathi A, Haslett R, Marsh IB. Strabismus surgery: Adjustable good for all? Eye, 2003; 17: 739-742.

4. Awadein A, Sharma M, Bazemore GM, Saeed HA, Guyton DL. Adjustable suture strabismus surgery in infants and children. J AAPOS. 2008; 12: 585-590.

5. Sharma P, Julka A, Gadia R, Chhabra A, Dehran M. Evaluation of single-stage adjustable strabismus surgery under conscious sedation. Indian J Ophthalmol. 2009; 57 (2): 121-125.

6. Nadeem S, Naeem BA, Khan F. Adjustable strabismus surgery: An early glance. Pak J Ophthalmol. 2018; 34 (2): 89-97.

7. Seijas O, de Liao GP, Merino P, Roberts CJ, Gómez de Liao R. Topical anesthesia in strabismus surgery: a review of 101 cases. J Pediatr Ophthalmol Strabismus, 2009; 46 (4): 218-222.

8. Kekunnaya R, Mendonca T, Sachdeva V. Pattern strabismus and torsion needs special surgical attention. Eye (London). 2015; 29 (2): 184-190.

9. Deschler EK, Irsch K, Guyton KL, Guyton DL. A new, removable, sliding noose for adjustable-suture strabismus surgery. J AAPOS. 2013; 17 (5): 524-527.
10. Coats DC, Olitsky SE. Strabismus surgery and its complications. Springer: Berlin, 2007. P: 37-39.

11. Yanoff M, Duker JS. Ophthalmology. Third Edition. Mosby: St. Louis, 2009; p 1331-1338.

12. Lueder GT, Archer SM, Hered RW, Karr DJ, Kodsi SR, Kraft SP, et al. Pediatric Ophthalmology and Strabismus. Section 6. Basic and Clinical Science Course. American Academy of Ophthalmology. San Francisco. 2015-2016; p 131-182.

13. Wong-Baker FACES Pain Rating Scale (Internet) Wikipedia Contributors. Wikipedia, The Free Encyclopedia; 4 December 2018 Available from: https://en.wikipedia.org/w/index.php?title=WongBaker_Faces_Pain_Rating_Scale\&oldid=871901004 Accessed: 30 April 2019.

14. Numeric Pain Rating Scale (Internet) Physiopedia contributors. Physiopedia; 26 April 2019. Available from:https://www.physiopedia.com/index.php?title=Nu meric_Pain_Rating_Scale\&oldid=209667. Accessed: 30 April 2019

15. Proxymetacaine Wikipedia, The Free Encyclopedia; Available from:

https://en.wikipedia.org/w/index.php?title= Proxymetacaine $\&$ oldid $=830475900$. Accessed: 1 May 2019.

16. Mazlow ML, Fletcher J. Selection of patients and results of 25 years of topical anesthesia and adjustable suture surgery. Am Orthopt J. 2013; 63 (1): 85-91.

17. Park JM, Lee SJ, Choi HY. Intraoperative adjustable suture strabismus surgery under topical and sunconjunctival anesthesia. Ophthalmic Surg Lasers Imaging, 2008; 39 (5): 373-378.

18. Hakim OM, El-Hag YG, Haikal MA. Strabismus surgery under augmented topical anesthesia. J AAPOS. 2005; 9 (3): 279-284.

19. Santhan KSG, Kelkar JA, Arora ER. Our experience with strabismus surgery under topical anesthesia performed at a tertiary care eye center. Indian J Ophthalmol. 2018; 66 (2): 342-343.

20. Vallés-Torres J, Garcia-Martin E, FernándezTirado FJ, Gil-Arribas LM, Pablo LE, Pea-Calvo P. Contact topical anesthesia versus general anesthesia in strabismus surgery. Arch Soc ESP Oftalmol. 2016; 91 (3): 108-113.

21. Modabber M, Dan AF, Coussa RG, Flanders $M$. Retrobulbar anaesthesia for adjustable strabismus surgery in adults: a prospective observational study. Can J Ophthalmol. 2018; 53 (6): 621-626.

\section{Author's Designation and Contribution}

Dr. Sana Nadeem; Assistant professor: Concept and Study Design, Drafting of Manuscript, Data Collection \& Analysis, Final review

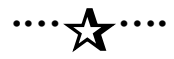

